\title{
Herausforderungen der Globalisierung für die Wirtschaftsinformatik-Ausbildung
}

Die Autoren
Hans Ulrich Buhl
Wolfgang König
Prof. Dr. Hans Ulrich Buhl
Universität Augsburg
Lehrstuhl für Betriebswirtschaftslehre,
Wirtschaftsinformatik
\& Financial Engineering
Universitätsstraße 16
86135 Augsburg
hans-ulrich.buhl@wiwi.uni-augsburg.de
Prof. Dr. Wolfgang König
Universität Frankfurt
Institut für Wirtschaftsinformatik
Mertonstraße 17
60054 Frankfurt am Main
koenig@wiwi.uni-frankfurt.de

Aristoteles wies der Menschheit vor über 2300 Jahren den richtigen Weg, dass die Erde eine Kugel ist. Heute behauptet Friedman, dass sie zunehmend flacher und damit zur Scheibe wird. Als Gründe nennt er: Die Globalisierungswelle wurde nach dem Fall der Mauer durch technische Entwicklungen weiter vorangetrieben und bahnte sich durch Workflow-Management-Systeme und Breitbandinternet ihren Weg nach Asien und Südamerika. Durch diese stark vereinfachten Kommunikationswege (wie man sie beispielsweise von flachen Unternehmensstrukturen her kennt) sind Outsourcing und Offshoring inzwischen die großen Stichworte, welche die Welt wieder flach erscheinen lassen. Es wird also immer einfacher, nicht nur „die IT“, sondern ganze Geschäftsprozesse ins Ausland zu verlagern. Man wird leichter ersetzbar - außer man besitzt besondere Wissensvorsprünge in diesem Standortwettbewerb, gehört zu den Spitzenkräften, besitzt Fach- und Führungskompetenzen, die auch heute noch im Weltmaßstab knapp sind. Was bedeutet das für unsere Gesellschaft, für unsere Wirtschaft, für unsere Ausbildung allgemein und speziell für die Wirtschaftsinformatik?

In einer derartigen flachen Welt wird die Fähigkeit zum Management globaler Wertschöpfungsnetzwerke immer wichtiger. Dabei genügt es nicht, über Größen- und Arbitragevorteile oder die entstehenden Kosten der Geschäftsabwicklung und Differenzierung bestens informiert $\mathrm{zu}$ sein, sondern auch die Kenntnisse der institutionellen Bedingungen, der Spezifika der Personalmärkte und nicht zuletzt der kulturellen Bedingungen sind von besonderer Bedeutung. Fragen, die es an dieser Stelle zu beantworten gilt, sind beispielsweise: Welche staatlichen Regulierungen gibt es? Wie sieht die Ausbildung und Arbeitsqualität der Arbeitskräfte aus? Wie hoch sind die Arbeitskosten?

Die Computerwoche berichtete Ende März 2007 über eine Befragung von A. T. Kearney der 500 größten deutschen Unternehmen, nach der hierzulande bis 2011 120.000 IT-Arbeitsplätze abgebaut werden sollen. Interessant ist, dass in den Bereichen IT-Betrieb, Wartung und Entwicklung 145.000 Arbeitsplätze wegfallen, im Bereich IT-Management hingegen 25.000 neue Stellen entstehen sollen. Als Hauptgründe für diese Entwicklung werden verbesserte Effizienz und Offshoring genannt. Diese Ergebnisse untermauern den Bedarf an interdisziplinär ausgebildeten Spitzenkräften, die spezifisches WirtschaftsinformatikKnow-how mit tiefem betriebswirtschaft- lichen Wissen und Managementfähigkeiten verbinden. Wie vielen Unternehmen fehlt es an starken Führungspersönlichkeiten, welche - wenn notwendig - auch gegen den Strom schwimmen und Perspektiven vermitteln können? Die große Bedeutung dieser Spitzenkräfte verdeutlicht auch das Beispiel Toll Collect: Erste Versuche sind gnadenlos gescheitert - mit enormem volkswirtschaftlichem Schaden. Erst nach Verstärkung des Entwicklungsteams durch Topleute wurde das Projekt letztlich zu einem Erfolg (Finanzen, Arbeitsplätze, Export, ...). Dieses Beispiel verdeutlicht außerdem, dass man - wie Peter Mertens in einem Interview der WIRTSCHAFTSINFORMATIK antwortete - „die IT-Branche nicht losgelöst von der gesamt-wirtschaftlichen, ja der gesamt-gesellschaftlichen Situation sehen darf“. Es kommt uns als Wirtschaftsinformatikern eine besondere Verantwortung zu, unsere Studenten heute auf die besonderen Herausforderungen von morgen vorzubereiten.

Dabei müssen wir uns der Vorteile der deutschen Wirtschaftsinformatik - sowohl im deutschsprachigen Bereich im Vergleich $\mathrm{zu}$ benachbarten disziplinär orientierten Studiengängen der Wirtschaftswissenschaften und der Informatik als auch im internationalen Vergleich zu unserer Schwesterdisziplin Information Systems - bewusst sein. Wir müssen unsere Alleinstellungsmerkmale - wie den hohen Stellenwert der Praxisrelevanz unserer Forschung - gezielt weiterentwickeln, damit sie auch im Vergleich zur amerikanischen - oft auf empirischem Rigour mit nachrangiger Relevance basierenden - IS-Forschung künftig weit erfolgreicher ist, besser die kommenden Herausforderungen annimmt und unsere Absolventen besser vorbereitet.

Die deutsche Hochschulausbildung in Wirtschaftsinformatik kann als klarer Standortvorteil gesehen werden. Doch um diesen zu sichern und weiter auszubauen, 
müssen wir nicht nur auf derzeitige Veränderungen reagieren, sondern im Vorfeld Entwicklungen wie sie in der o.g. Studie prognostiziert werden, bereits heute in unserer Ausbildung berücksichtigen. Dabei ist es nicht ausreichend, neue Inhalte zu identifizieren und zusätzlich anzubieten, sondern es ist notwendig, das gesamte Lehrkonzept weiterzuentwickeln und die relevanten Inhalte zu selektieren. Nur dann werden sich unseren Absolventen auch morgen noch die hervorragenden $\mathrm{Zu}$ kunftschancen bieten, wie wir diese heute überall beobachten können: Interessanterweise haben nach allen uns vorliegenden aktuellen Studien Wirtschaftsinformatiker und Wirtschaftsingenieure nicht nur weit bessere Berufschancen als Wirtschaftswissenschaftler, sondern selbst im reinen IT-Bereich weit bessere als z. B. Informatiker. Damit das auch morgen so ist, müssen unsere Absolventen noch mehr auf die kundennahen Aufgaben vorbereitet werden und auf das Management internationaler Projekte und internationaler Wertschöpfungsnetzwerke. Mit weltweit üblichen Softwareentwicklungsfähigkeiten haben wir bei unserem Lohnniveau im globalen Wettbewerb dagegen keine Chance. Das wird an unseren Hochschulen - aber nicht nur dort - noch viel zu wenig verstanden. Wir müssen bei diesen Anpassungen Vorreiter sein und unsere Praxisnetzwerke noch mehr dazu nutzen, diese $\mathrm{Zu}$ kunftsperspektiven in der Öffentlichkeit und speziell bei Abiturienten bekannter zu machen.

Bei dieser marktorientierten Weiterentwicklung unserer Studiengänge müssen wir viele Hemmnisse überwinden. Waren in der Diplomwelt HRK/KMK-verordnete Stundenbegrenzungen zu überwinden, plagen uns jetzt in den Bachelor-/Master-Studiengängen die Akkreditierungsstandards, bei denen man oft nicht sauber zwischen Mindest- und Höchstanforderungen unterscheidet. Eine Arbeitsbelastung unserer Studenten von 1.800 Arbeitsstunden p. a. (=60 ECTS-Credits) ist als Mindeststandard völlig in Ordnung - als Höchststandard sicher nicht. Mit der Gewöhnung der Studenten an eine 35-Stundenwoche werden wir die o. g. Herausforderungen sicher nicht bewältigen. Integrationsstudiengänge wie Wirtschaftsinformatik erfordern eine deutlich höhere Stundenanzahl als dies bei den disziplinären Studiengängen der Fall ist, da sich diese Studenten in jeder der beteiligten Disziplinen in Konkurrenz zu Studierenden der Einzeldisziplinen bewähren müssen. Gerade für wettbewerbskritische Fach- und Führungsaufgaben sind solche interdisziplinären Kenntnisse von ebenso hoher Bedeutung wie die Erfahrungen in mehrfach fordernden Studiengängen. Es kann also nicht sein, dass die Möglichkeiten, Studenten $\mathrm{zu}$ fordern und gleichzeitig zu fördern, durch solche Standards nach oben begrenzt werden.

Des Weiteren entfachte der Bologna-Prozess heftige Diskussionen um das internationale Ansehen der deutschen Hochschulausbildung in Folge der Anpassung an das Bachelor-/Master-System. Auch wenn die damit verbundenen nationalen Akkreditierungsrichtlinien (aufgrund der Empfehlungen der GI und WKWI) keine nachhaltigen Einschränkungen zur Folge haben, so bleibt dennoch zu bedenken, dass sowohl bei diesen als auch bei internationalen Akkreditierungen das Problem entstehen kann, dass die notwendige Flexibilität unserer Studiengänge durch zu starre Anwendung von Akkreditierungsrichtlinien eingeschränkt wird. Und was nutzt ein international akkreditierter Abschluss, wenn wir uns nicht mehr auf die gewachsenen Stärken vor Ort konzentrieren und diese ausbauen können? Qualitätssicherung in Lehre und Forschung ist natürlich - auch im internationalen Vergleich - notwendig, aber die absolute Förderung von Lehre und Forschung am Standort muss Priorität haben und darf nicht eingeschränkt werden. Darüber hinaus stellt sich die Frage: Wie können wir uns jetzt für die Herausforderungen von morgen rüsten, wenn wir „alten“ Vorgaben gerecht werden müssen?

Betrachtet man (1) die Entwicklung der Studentenzahlen, (2) die demografische Veränderung und (3) den kommenden Bedarf an Spitzenkräften in Deutschland, so wird schnell klar, welche Herausforderungen auf die Ausbildung zukommen und warum an Off- / Nearshoring letztendlich kein Weg vorbei führt.

(1) Aufgrund der Volatilität bei IT-Arbeitsplätzen sind Abiturienten und deren Eltern verunsichert - dies spiegelt sich in den Einschreibungszahlen für Informatik sowohl in Deutschland als auch in den USA wider - dort auch in unserer Schwesterdisziplin Information Systems. Waren diese bis 2001 noch stark zunehmend, so sind sie seit dem Dotcom-Crash stark rückläufig. Im Gegensatz dazu sind die Einschreibungszahlen für Wirtschaftsinformatik zwar stabil, jedoch nicht im erforderlichen Maße wachsend und scheinen sich bei rund 2/3 der Informatik eingependelt zu haben.

(2) Den Einschreibungszahlen stehen zudem in den letzten Jahren leicht stei- gende (Fach-) Abiturientenzahlen gegenüber, die sich annähernd analog $\mathrm{zu}$ der Anzahl der 18- bis 20-Jährigen entwickelt haben. Laut Statistischem Bundesamt wird die Größe dieser Altersgruppe jedoch von derzeit ca. 1,95 Mio. bereits bis 2010 um knapp $11 \%$ und bis 2020 um $22 \%$ sinken. Auch wenn die Wirtschaftsinformatik inzwischen zu den Top 20 der studentenstärksten Studiengänge zählt (bei Männern sogar Top 11), muss unser Studiengang bei einer angenommenen proportionalen Abnahme der Abiturientenzahlen dennoch wesentlich attraktiver gemacht werden, um die Einschreibungen zumindest konstant zu halten. Ein substanzielles Wachstum ist aber dringend erforderlich, wenn wir Märkte nicht aufgeben, Arbeitsplätze sichern und neue insbesondere im stark wachsenden Dienstleistungsbereich hierzulande schaffen wollen.

(3) Doch selbst bei Erreichen dieses ehrgeizigen Ziels wird es nicht möglich sein, den Bedarf an qualifizierten Absolventen für das IT-Management vollständig aus dem Inland zu decken. Bereits seit ein paar Jahren mangelt es in Deutschland an qualifizierten IT-Fachund -Führungskräften. Statistiken zeigen weiterhin - trotz Off- und Nearshoring von Software und IT-Services - eine zunehmende Nachfrage in naher Zukunft. Zu bedenken bleibt dabei der - bereits genannte - dahingehende Trend, zwar IT-Arbeitsplätze der Bereiche Betrieb, Wartung und Entwicklung ins Ausland zu verlagern, jedoch (nicht zuletzt dadurch) notwendige Stellen im IT-Management im Inland $\mathrm{zu}$ schaffen. Und es ist insbesondere die Aufgabe der WirtschaftsinformatikStudiengänge, Studenten für diese Aufgaben auszubilden, da sie die nötige Verknüpfung der Unternehmensziele mit der Prozess- und IT-Gestaltung zum Gegenstand haben.

Was ist jetzt zu tun?

(1) Zunächst müssen wir sicherstellen, dass der Hochschulausbildung die nötige Flexibilität eingeräumt bleibt. Unseren Vorteil der hervorragenden Wirtschaftsinformatik-Ausbildung dürfen wir nicht einengen lassen. Es ist wichtig, den Absolventen von morgen sowohl solides Grundlagenwissen als auch die spezialisierten Methoden und Erkenntnisverfahren der deutschen Wirtschaftsinformatik zu vermitteln, um sie auf die 
kommenden IT-Management-Aufgaben vorzubereiten. Dabei sollte bereits jetzt der deutsche Bildungsbedarf der nächsten Jahre zugrunde liegen, insbesondere die Fähigkeit zum Management internationaler Wertschöpfungsnetzwerke. Und es ist wichtig, auch Freiräume für Experimente zu geben.

(2) Des Weiteren erscheint es sinnvoll, Public-Private-Partnerships zwischen künftigen Arbeitgebern und Hochschulen als Signalwirkung zu forcieren. Man könnte sich auch einen Austausch von Wissenschaftlern und Praktikern vorstellen - beispielsweise Wissenschaftler ein Jahr in der Praxis und umgekehrt. Beides Möglichkeiten, um die beiden „Welten“ stärker zu vernetzen, wodurch sowohl die immer wichtiger werdende Praxisnähe der Ausbildung als auch der Transport der Forschung in die Praxis gefördert wird. Ebenso brauchen wir vorausschauende Praktiker, die aufkommende Frage- und Problemstellungen in kurzer Zeit in die Hochschulen einbringen und damit gemeinsam mit den Hochschullehrern dafür sorgen, dass genügend Absolventen für den Bedarf von morgen ausgebildet werden.

(3) Eine weitere Herausforderung an die Hochschulausbildung sind Konzepte zur besonderen Förderung und Forderung der Topstudenten. Diese könnten beispielsweise in der Übernahme von Studiengebühren oder besonderen/spezialisierten Praktikaangeboten bis hin zu einer Jobgarantie liegen. Hierbei ist ebenfalls der spezielle Aufruf an die Praxis notwendig, derartige Kooperationen mit den Hochschulen zu schaffen.
(4) Natürlich ist es darüber hinaus notwendig, dass die Politik die notwendigen Veränderungen an den Hochschulen unbürokratisch unterstützt. So ist es ebenso naheliegend wie wirtschaftlich sinnvoll, die neuen Studienplätze die es zu schaffen gilt - insbesondere in Integrationsstudiengängen wie der Wirtschaftsinformatik zu schaffen.

Es bedarf also der Anstrengungen aller Stakeholder der gesamten Hochschullandschaft, die für eine erfolgreiche Hochschulausbildung von Bedeutung sind: Viele Joint-Ventures zwischen Wirtschaft, Wissenschaft und Politik auf nationaler wie internationaler Ebene sind erforderlich, um die skizzierten Herausforderungen zu bewältigen.

Prof. Dr. Hans Ulrich Buhl Prof. Dr. Wolfgang König

\section{In eigener Sache}

Die Herausgeber unserer Zeitschrift üben ihr Amt für eine begrenzte Zeit aus (maximal 13 Jahre). Endet eine Mitgliedschaft im Herausgeberkreis, bestimmt das Gremium neue Mitglieder durch geheime Wahl. Wir begrüßen als neuen Herausgeber Herrn Prof. Dr. Detlef Schoder, Universität zu Köln, und freuen uns auf eine spannende und erfolgreiche gemeinsame Arbeit.

Herausgeber, deren Amtszeit 13 Jahre überschritten hat und die der Zeitschrift weiterhin aufs Engste verbunden bleiben, werden künftig mit dem Klammerzusatz „langjähriger Hrsg." in der Liste der Herausgeber aufgeführt.

Hans Ulrich Buhl Wolfgang König Geschäftsführende Herausgeber 